\title{
Riemann's Hypothesis and Conjecture of Birch and Swinnerton-Dyer are False
}

Chun-Xuan Jiang

P. O. Box 3924, Beijing 100854 China

jcxuan@sina.com

\begin{abstract}
All eyes are on the Riemann's hypothesis, zeta and L-functions, which are false, read this paper. The Euler product converges absolutely over the whole complex plane. Using factorization method we can prove that Riemamn's hypothesis and conjecture of Birch and Swinnerton-Dyer are false. All zero computations are false, accurate to six decimal places. Riemann's zeta functions and $L$ - functions are useless and false mathematical tools. Using it one cannot prove any problems in number theory. Euler totient function $\phi(n)$ and Jiang's function $J_{n+1}(\omega)$ will replace zeta and $L$ - functions.
\end{abstract}

\section{Introduction}

The function $\zeta(s)$ defined by the absolute convergent series

$$
\zeta(s)=\sum_{n=1}^{\infty} \frac{1}{n^{s}}
$$

in complex half-plane $\operatorname{Re}(s)>1$ is called the Riemann's zeta function.

The Riemann's zeta function has a simple pole with the residue 1 at $s=1$ and the function $\zeta(s)$ is analytically continued to whole complex plane. We then define the $\zeta(s)$ by the Euler product

$$
\zeta(s)=\prod_{P}\left(1-P^{-s}\right)^{-1}
$$

where the product is taken all primes $P, s=\sigma+i t, i=\sqrt{-1}, \sigma$ and $t$ are real.

The Rieman's zeta function $\zeta(s)$ has no zeros in $\operatorname{Re}(s)>1$. The zeros of $\zeta(s)$ in $0<\operatorname{Re}(s)<1$ are called the nontrivial zeros. In $1859 \mathrm{G}$. Riemann conjectured that every zero of $\zeta(s)$ would lie on the line $\operatorname{Re}(s)=1 / 2$. It is called the Riemenn's hypothesis. [1] We have

$$
\zeta(s=\sigma+i t, \sigma \geq 1) \neq 0
$$

We define the elliptic curve [2]

$$
E_{D}: y^{2}=x^{3}-D^{2} x
$$

where $D$ is the congruent number.

Assume that $D$ is square-free. Let $P$ be a prime number which does not divide $2 D$. Let $N_{P}$ denote the numbers of pairs $(x, y)$ where $x$ and $y$ run over the integers modulo $P$, 
which satisfy the congruence

$$
y^{2} \equiv x^{3}-D^{2} x \bmod P .
$$

Put

$$
a_{P}=P-N_{P}
$$

We then define the $L$-function of $E_{D}$ by the Euler product

$$
L\left(E_{D}, s\right)=\prod_{(P, 2 D)=1}\left(1-a_{P} P^{-s}+P^{1-2 s}\right)^{-1}
$$

where the product is taken over all primes $P$ which do not divide $2 D$. The Euler product converges absolutely over the half plane $\operatorname{Re}(s)>3 / 2$, but it can be analytically continued over the whole complex plane. For this function, it is the vertical line $\operatorname{Re}(s)=1$ which plays the analogue of the line $\operatorname{Re}(s)=1 / 2$ for the Riemann zeta function and the Dirichlet $L$ - functions. Of course, we believe that every zero of $L\left(E_{D}, s\right)$ in $\operatorname{Re}(s)>0$ should lie on the line $\operatorname{Re}(s)=1$. It is called a conjecture of Birch and Swinnerton-Dyer (BSD). We have

$$
L\left(E_{D}, s=\sigma+i t, \sigma \geq 3 / 2\right) \neq 0
$$

\section{Riemann's Hypothesis is false}

Theorem 1. Euler product converges absolutely in $\operatorname{Re}(s)>1$. Let $s_{0}=1 / 2+$ it, using factorization method we have

$$
\zeta\left(s_{0}=1 / 2+i t\right) \neq 0
$$

Proof. Let $s=2 s_{0}, 2.2 s_{0}, 2.8 s_{0}, 3 s_{0}, 4 s_{0}, 5 s_{0}, \cdots P_{0} s_{0}$

We have the following Euler product equations

$$
\begin{aligned}
& \zeta\left(2 s_{0}\right)=\zeta\left(s_{0}\right) \prod_{P}\left(1+P^{-s_{0}}\right)^{-1} \neq 0, \\
& \zeta\left(2.2 s_{0}\right)=\zeta\left(s_{0}\right) \prod_{P}\left(P^{-1.2 s_{0}}+\frac{1-P^{-1.2 s_{0}}}{1-P^{-s_{0}}}\right)^{-1} \neq 0, \\
& \zeta\left(2.8 s_{0}\right)=\zeta\left(s_{0}\right) \prod_{P}\left(P^{-1.8 s_{0}}+\frac{1-P^{-1.8 s_{0}}}{1-P^{-s_{0}}}\right)^{-1} \neq 0, \\
& \zeta\left(3 s_{0}\right)=\zeta\left(s_{0}\right) \prod_{P}\left(1+P^{-s_{0}}+P^{-2 s_{0}}\right)^{-1} \neq 0, \\
& \zeta\left(4 s_{0}\right)=\zeta\left(s_{0}\right) \prod_{P}\left(1+P^{-s_{0}}\right)^{-1} \prod_{P}\left(1+P^{-2 s_{0}}\right)^{-1} \neq 0,
\end{aligned}
$$




$$
\begin{aligned}
& \zeta\left(5 s_{0}\right)=\zeta\left(s_{0}\right) \prod_{P}\left(1+P^{-s_{0}}+P^{-2 s_{0}}+P^{-3 s_{0}}+P^{-4 s_{0}}\right)^{-1} \neq 0, \\
& \quad \cdots \cdots \\
& \zeta\left(P_{0} s_{0}\right)=\zeta\left(s_{0}\right) \prod_{P}\left(1+\cdots+P^{-\left(P_{0}-1\right) s_{0}}\right)^{-1} \neq 0,
\end{aligned}
$$

Since the Euler product converges absolutely in $\operatorname{Re}(s)>1$, the equation (10)-(16) are true.

From (10)-(16) we obtain

$$
\zeta\left(s_{0}\right) \neq 0
$$

All zero computations are false and approximate, accurate to six decimal places. Using three methods we proved the $\mathrm{RH}$ is false [3]. Using the same Method we are able to prove that all Riemann's hypotheses also are false. All $L-$ functions are false and useless for number theory.

\section{The Conjecture of Birch and Swinnerton-Dyer is false.}

Theorem 2. Euler product converges absolutely in $\operatorname{Re}(s)>3 / 2$. Let $s_{1}=1+i t$. Using factorization method we have

$$
L\left(E_{D}, S_{1}=1+i t\right) \neq 0
$$

Proof. Let $s=2 s_{1}, 3 s_{1}, 4 s_{1}, \cdots$

we have the following Euler product equations.

$$
\begin{aligned}
& L\left(E_{D}, 2 s_{1}\right)=L\left(E_{D}, s_{1}\right) \prod_{(P, 2 D)=1}\left(P^{-2 s_{1}}+\frac{1-\left(a_{P}+1\right) P^{-2 s_{1}}+a_{P} P^{-3 s_{1}}}{1-a_{P} P^{-s_{1}}+P^{1-2 s_{1}}}\right)^{-1} \neq 0 \\
& L\left(E_{D}, 3 s_{1}\right)=L\left(E_{D}, s_{1}\right) \prod_{(P, 2 D)=1}\left(P^{-4 s_{1}}+\frac{1-a_{P} P^{-3 s_{1}}-P^{-4 s_{1}}+a_{P} P^{-5 s_{1}}}{1-a_{P} P^{-s_{1}}+P^{1-2 s_{1}}}\right)^{-1} \neq 0 \\
& L\left(E_{D}, 4 s_{1}\right)=L\left(E_{D}, s_{1}\right) \prod_{(P, 2 D)=1}\left(P^{-6 s_{1}}+\frac{1-a_{P} P^{-4 s_{1}}-P^{-6 s_{1}}+a_{P} P^{-7 s_{1}}}{1-a_{P} P^{-s_{1}}+P^{1-2 s_{1}}}\right)^{-1} \neq 0
\end{aligned}
$$

Since the Euler product converges absolutely in $\operatorname{Re}(s)>3 / 2$, equations (18)-(20) are true.

From (18)-(20) we obtain

$$
L\left(E_{D}, s_{1}\right) \neq 0
$$

All zero computations are false and approximate. Using the same method we are able to prove all $L(E, s) \neq 0$ in whole complex plane.

The elliptic curves are not related with the Diophantine equations and number theory [4]. Frey and Ribet did not prove the link between the elliptic curve and Fermat's equation [4,5]. Wiles proved Taniyama-Shimura conjecture based on the works of Frey, Serre, Ribet, Mazuer and Taylor, which have nothing to do with Fermat's last theorem [6]. "Taniyama-Shimura conjecture" was in obscurity for about 20 years till people seriously started thinking about elliptic curves. 
Mathematical proof does not proceed by personal abuse, but by show careful logical argument. Wiles proof of Fermat's last theorem is false [7-9]. In 1991 Jiang proved directly Fermat's last heorem [10,11].

\section{Conclusion.}

The zero computations of zeta functions and $L$ - functions are false. Riemann's zeta functions and $L$ - functions are useless and false mathematical tools. Using it one cannot prove any problems in number theory [12]. The heart of Langlands program(LP) is the $L$-functions [13]. Therefore LP is false. Wiles proof of Fermat last theorem is the first step in LP. Using LP one cannot prove any problems in number theory, for example Fermat's last theorem [6]. Euler totient function $\phi(n)$ and Jiang's function $J_{n+1}(\omega)$ will replace Riemann's zeta functions and $L$ - functions [13-15].

\section{References}

[1] B. Riemann, Uber die Anzahl der Primzahlen under enier gegebener GrÖsse, Monatsber. Akad. Berlin. 671-680 (1859)

[2] John Coates, Number theory, Ancient and Modern, In: ICCM 2007. vol. I, 3-12.

[3] Chun-Xuan Jiang, Disproofs of Riemann's hypothesis. Algebras, Groups and Geometries, 22, 123-136(2005) http://www.i-b-r.org/docs/JiangRiemann.pdf; http://vixra.org/abs/1004.0028; http://www.wbabin.net/math/xuan9.pdf

[4] G. Frey, Links between stable elliptic curves and certain diophantine equations, Annales Universitatis Saraviensis 1 (1986), 1-40.

[5] K. A. Ribet, On modular representations of Gal $(\bar{Q} / Q)$ arising from modular forms, Invent. Math. 100 (1990), 431-476.

[6] A. Wiles, Modular elliptic curves and Fermat's last theorem, Ann. of Math. 141(1995), 443-551.

[7] G. Perelman, Perelman disproves Wiles proof of Fermat's theorem. http://journalism.cedar-falls.k12.ia.us/pictures/4.13/entzarticle.pdf. http://documentsearch.org/pdf/perelman-disproves-wiles-proof-of

[8] Y. G. Zhivotov, Fermat's last theorem and Kenneth Ribet's mistakes, http://www.wbabin.net/files/4438_zhivotov.pdf; http://newrotor.narod.ru/english. html.

[9] Y. G. Zhivotov, Fermat's last theorem and mistakes of Andrew Wiles, http://www.newrotor.narod.ru/fermat.html http://www.readfree.net/bbs/read.php?tid=4462593

[10] Chun-Xuan Jiang, Automorphic function and Fermat's last theorem (1) http://www.wbabin.net/ntham/xuan167.pdf http://www.vixra.org/pdf/1012.0007v1.pdf

[11] Chun-Xuan Jiang, Automorphic function and Fermat's last theorem (3)(Fermat's proof of FLT), http://www.wbabin.net/ntham/xuan169.pdf http://www.vixra.org/pdf/1012.0009v1.pdf

[12] Arthur, et al., editors, On certain $L$ - functions. AMS, CMI. 2011. volume13.

[13] S. Gelbart, An elementary introduction to the Langlands program, Bull, of AMS. 10(1984) 
177-219.

[14] Chun-Xuan Jiang, The Hardy-Littlewood prime $k$ - tuple conjecture is false.

(http://www.wbabin.net/math/xuan77.pdf); (http://vixra.org/pdf/1003.0234v1.pdf)

[15] Chun-Xuan Jiang, Jiang's function $J_{n+1}(\omega)$ in prime distribution,

(http://www.wbabin.net/math/xuan2.pdf) (http://www.vixra.org/pdf/0812.0004v2.pdf)

[16] Chun-Xuan Jiang, Foundations of Santilli's isonumber theory with applications to new cryptograms, Fermat's theorem and Goldbach's conjecture, Inter. Acad. Press, 2002. MR2004c:/11001.

(http://www.i-b-r.org/docs/jiang.pdf); (http://www.wbabin.net/math/xuan13.pdf)

\section{最后说明}

Euler product 是绝对收敛的。方程（10）-（16）和（18）-（20）是绝对正确的。20 世纪数论上没有重大突破, 主要大家集中力量研究黎曼假设 (RH)。利用 $\mathrm{RH}$ 来研究数论问 题。 RH 所有零点计算都是错误的, 因为无穷 级数无法计算精确值, 这些错误零点计算使 100 年来所有数学家都相信 $\mathrm{RH}$ 是正确的, 顶尖数学家都集中力量证明 $\mathrm{RH}$, 并指出 $\mathrm{RH}$ 是 21 世纪最大要解决问题。所有 RH 专家都集中在 IAS。Institute of advanced study was the undisputed Mecca of the Riemann hypothesis. 20 世纪所有伟大数学家都研究 RH。例如 Hibert. Hardy, Weil, ... If I were to awaken after having stept for five hundred years, my first question would be: Has the Riemann hypothesis been proven? (David Hibert)。如果没有蒋春暄否定 RH, 那末再过 500 年 $\mathrm{RH}$ 仍不能解决. $\mathrm{RH}$ 是数论的基础。利用 $\mathrm{RH}$ 许多数学家证明上千个定理, 这些定理都是错的。 RH 零点是错的, 那末 $L-$ functions 零点也是错的。利用 zeta 函数和 $L-$ functions 不能证明数论中任何问题。

从 $\mathrm{RH}$ 出发利用 $L-$ 函数和椭圆曲线 R. L. Langlands 提出 Langlands program(LP)。把许 多没有关系数学问题统一联系起来。利用中国成语 “张冠李戴” 办法解决所有数学问题, 怀 尔斯证明费马大定理就是 LP 第一次应用最大成果, 怀尔斯失败, 身败名裂, 也是 LP 失败。 蒋春暄否定 RH 在 AIM, CLAYMA, IAS, THES, MPIM, MSRI 已无人研究 RH 和 数论。但是他们仍抓住 $L-$ 函数和椭圆曲线不放继续研究, 所以他们集中力量研究 BSD 猜 想。目前国内外数论专家下岗或改行。但他们对蒋春暄开创数论新时代, 他们都不讲话, 因 为中国对蒋春暄数论成果不承认, 定为伪科学。

以 RH 开创数论时代已结束。一个新的数论时代将开始。 